Understanding Wind Ramp Events Through Analysis of Historical Data

C. Kamath

September 1, 2009

IEEE PES Transmission and Distribution Conference and Expo

New Orleans, LA, United States

April 20, 2010 through April 22, 2010 
This document was prepared as an account of work sponsored by an agency of the United States government. Neither the United States government nor Lawrence Livermore National Security, LLC, nor any of their employees makes any warranty, expressed or implied, or assumes any legal liability or responsibility for the accuracy, completeness, or usefulness of any information, apparatus, product, or process disclosed, or represents that its use would not infringe privately owned rights. Reference herein to any specific commercial product, process, or service by trade name, trademark, manufacturer, or otherwise does not necessarily constitute or imply its endorsement, recommendation, or favoring by the United States government or Lawrence Livermore National Security, LLC. The views and opinions of authors expressed herein do not necessarily state or reflect those of the United States government or Lawrence Livermore National Security, LLC, and shall not be used for advertising or product endorsement purposes. 


\title{
Understanding Wind Ramp Events Through Analysis of Historical Data
}

\author{
Chandrika Kamath, Senior Member, IEEE
}

\begin{abstract}
As renewable resources start providing an increasingly larger percentage of our energy needs, we need to improve our understanding of these intermittent resources so we can manage them better. In the case of wind resources, large unscheduled changes in the energy output, called ramp events, make it challenging to keep the load and the generation balanced. In this paper, we show that simple statistical analysis of the wind energy generation can provide quantitative insights into these ramp events. In particular, this analysis can help answer questions such as the time period during the day when these events are likely to occur, the relative severity of positive and negative ramps, and the frequency of their occurrence.
\end{abstract}

Index Terms-wind energy, ramp events, statistical analysis.

\section{INTRODUCTION}

$\mathbf{T}$ HERE is an increasing interest in renewable sources of energy, such as wind and solar, as a result of various climate change initiatives and a desire for energy independence from fossil fuels. However, the intermittent nature of these sources can make them difficult to manage. In addition, for renewables such as wind, the occurrence of ramp events, where the energy generated suddenly increases or decreases rapidly in response to changes in wind velocity, can lead to challenges in keeping the load and generation balanced at all times.

In the past, the percentage of energy from wind sources, relative to the peak load, was small. For example, in 2006, the California Independent System Operator (CaISO) managed over 2200 MW of wind generation, which was only $4 \%$ of the total generation resources in the area [1]. The Tehachapi area in Southern California, which is one of the largest wind generation areas under CaISO, had 740MW installed capacity. At this capacity, the ramp events are relatively small. So, it is relatively easy to keep the load balanced, and the generation required to back up wind power is small as well.

However, with increasing wind penetration, the size of the ramp events has also increased. For example, the installed wind capacity in the Bonneville Power Administration (BPA) is currently over $2000 \mathrm{MW}$ and is expected to increase to over $3000 \mathrm{MW}$ by 2010 , when it will be $30 \%$ of the peak load. At this capacity, the wind ramps can be quite large, changing by nearly $1000 \mathrm{MW}$ in an hour, and it becomes more of a challenge to balance the load and the generation. The control room operators and schedulers now have to monitor the wind generation more closely, and plan for enough backup generation to meet the load, especially during downward ramp events when there is an unscheduled decrease in wind generation by a large amount in a short time.

C. Kamath is with the Lawrence Livermore National Laboratory, Livermore, CA 94551 USA e-mail: kamath2@llnl.gov (see http://people.1lnl.gov/kamath2).
There are several ways in which we can better manage this intermittent nature of wind resources. These include more accurate wind generation forecasts as well as an improved understanding of the ramp events. In this paper, we show that we can gain quantitative insights into ramp events by analyzing recent historical data on actual generation from wind farms. Specifically, simple statistics extracted from the data can answer questions, such as, do ramps occur more frequently in the mornings or evenings, do the negative ramps occur as frequently as the positive ramps and are they as severe, how should ramps be defined especially in light of increasing penetration, and do severe ramps occur rarely or are they relatively frequent?

This paper is organized as follows. First, in Section II, we describe the data used in the analysis, followed in Section III by details on the cleaning of the data, the definition of ramp events, the statistics extracted from the data, and our observations. We conclude with some ideas for future work.

\section{DATA DESCRIPTION}

We conduct our study of ramp events using actual wind generation data from BPA (www.bpa.gov) for the years 20072008. We chose data from the recent past as any analysis of these data is likely to be more relevant. Further, the last few years have seen a large increase in installed wind power in BPA balancing region, increasing from $700 \mathrm{MW}$ in 20062007 to over $1300 \mathrm{MW}$ in 2008 and more than $2000 \mathrm{MW}$ in 2009 [2], [3]. This increase in capacity occurs at various times during the year as new wind farms are connected to the grid.

The data available for the period 2007-2008 are the total generation from all the wind farms in the BPA region around the Columbia Basin [4]. The data are sampled at 5 minute intervals, and for each data point, include the time (year, month, day, hour and minute), the actual wind generation, and the total load at that time. Figure 1 shows the wind energy generated and the load for the first week of January 2008 for the BPA balancing area. Note the daily periodicity in load; however, there is no such periodicity in the wind energy generation. Figure 2 is a longer sequence for the wind energy for the month of January 2008. Note the intermittent nature of the energy generated, with some days having no generation, while on other days, it can reach as high as $1200 \mathrm{MW}$.

From July 2008 onward, the BPA data also include the wind energy scheduled by the operators at each data point; this is not considered in the present analysis.

Though our work focused on both 2007 and 2008 data, in this paper, we present the results only for 2008. The results for 2007 are very similar and will be available in a detailed technical report available from the author's web site. 


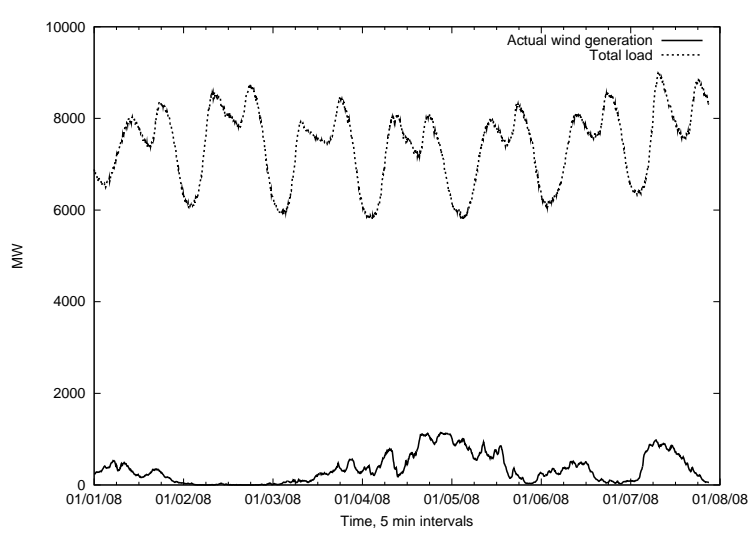

Fig. 1. The load (top curve) and the wind generation (bottom curve) for the first week of January 2008 for the BPA balancing area. Note the daily periodicity in the load curve.

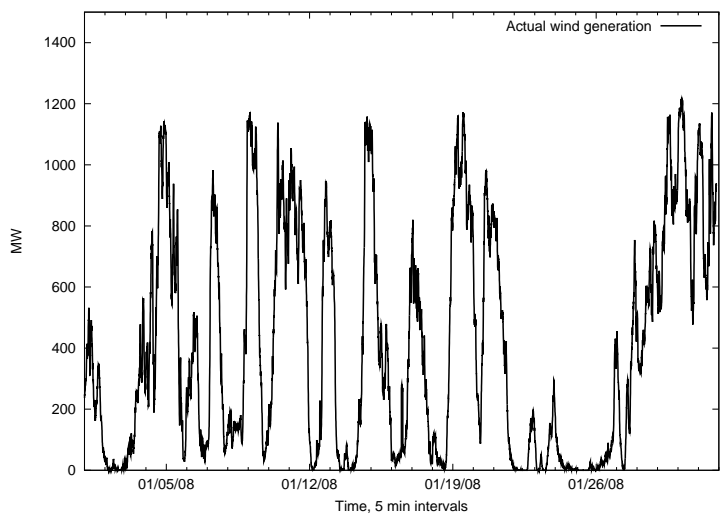

Fig. 2. The wind generation for January 2008 for the BPA balancing area. Note the intermittent nature of the energy generated.

The intent of the work presented in this paper is to show that we can gain insights into wind ramp events through simple statistical analysis of the wind generation data. However, we caution that the results of this preliminary analysis must be interpreted with care as they reflect the conditions for a specific year, 2008, and a specific region, namely, the Columbia Basin. We are conducting additional in-depth analysis of the data, especially over longer time periods, to determine if the observations made in this paper hold over the long term.

\section{ANALYSis OF THE DATA}

Our analysis of ramp events consists of three main steps: data cleaning, ramp definition, and extraction of various statistics. We next discuss each of these steps in more detail.

\section{A. Cleaning the data}

Thre are two main parts to cleaning the data. The first is to bring all the data to a consistent format, with each five minute interval described by four variables: the date (month, day, and year), the time (in hours and minutes), the actual wind generation, and the total load at that time.

The second step is to fill in the missing data. Sometimes, only one or two consecutive time intervals are missing, while in other cases, an hour or more of data may be missing. These missing values are represented as blanks in the original data. For our analysis, if only one or two values were missing, they were filled in via interpolation. If more than two consecutive values were missing, they were replaced by -9999 , and ignored in any further analysis.

\section{B. Defining a ramp event}

Once the data have been cleaned and brought to a consistent format, we need to identify the ramp events in the data. Though it is often easy to identify ramp events visually, there is no standard way in which such events are defined mathematically [5]. Therefore there are different ways in which we can interpret a "large increase or decrease in energy output in a short time." In this paper, we consider two definitions:

- Ramp definition 1: In this simple definition, a ramp event is considered to occur at the start of an interval if the magnitude of the increase or decrease in generation at a time $\Delta T$ ahead of the interval is greater than a predefined threshold, $\operatorname{Tr}$ :

$$
|M W(T+\Delta T)-M W(T)|>T r
$$

- Ramp definition 2: Since definition 1 focuses only on the end points of the interval being considered, it can miss ramp events if they occur between the two endpoints, though the endpoints themselves may not exhibit a large change in magnitude. Our second definition considers the minimum and maximum value of wind generation between the two endpoints (inclusive):

$$
\max (M W[T, T+\Delta T])-\min (M W[T, T+\Delta T])>T r
$$

Alternative definitions are also possible which are based on the average slope of the energy over a fixed time interval, say, 30 minutes. This reflects the visual perception of a ramp event as "steep gradients in power production" [5]. We can also use other descriptors, such as the duration of a ramp event, to characterize them [6]. Further, as the time series data are noisy, we can smooth them before identifying the ramp events; this is not done in the present work.

The ramp is considered a positive or negative ramp depending on whether the generation increases or decreases over time. Negative ramps are usually more challenging as the operators have to find other generation to replace the decrease in generation from wind sources and keep the load balanced.

For example, Figure 3 shows large positive and negative ramps around 3:45pm on June 21, 2008. From the hour prior, the generation increases by $667 \mathrm{MW}$ to a peak of $855 \mathrm{MW}$ and then drops by $577 \mathrm{MW}$ an hour later.

The time intervals, $\Delta T$, chosen for the ramp events are typically 15 minutes, 30 minutes, and 1 hour. The choice of the threshold $T r$ is more difficult. On one hand, we can set it to be an absolute value, say, $300 \mathrm{MW}$ for a $15 \mathrm{~min}$ ramp or $450 \mathrm{MW}$ for a $60 \mathrm{~min}$ ramp. This value could be system dependent and chosen to reflect the amount of energy that is difficult to procure in the given time interval to keep the system balanced. On the other hand, we can define the threshold as a 


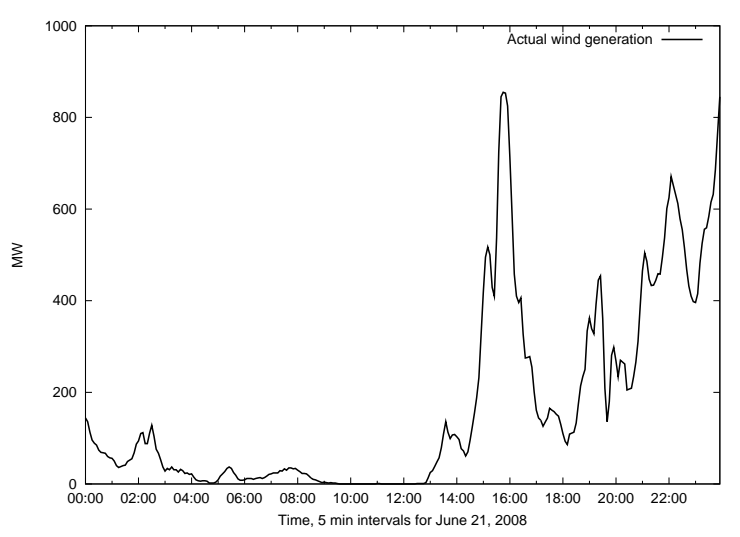

Fig. 3. The large positive and negative ramps in the hour just before and after 3:45pm on June 21, 2008.

percentage, say $20 \%$, of the installed nameplate capacity for a wind farm. The issue with this definition is that we need to know what the installed capacity is at any time. This may vary over time as existing turbines are upgraded or shut down for repairs or maintenance. In the latter case, we may see ramps of smaller magnitude, while in the former case, an event which qualified as a ramp event on one day, may not be a ramp event on another day with a higher nameplate capacity. However, the challenges of managing a ramp of that magnitude would be the same for both days.

In light of this, we chose a fixed threshold value for our analysis and experimented with various values to see how sensitive the results are to our choice of threshold. In practice, this threshold should be selected appropriately based on input from system operators and schedulers. It would likely be different for different regions and could vary over the years, evolving to match the changes in the generation mix at a utility.

\section{Statistics and observations on ramp events}

We next describe the various statistics obtained for the ramp events, followed by our observations on these statistics. First, we consider the change, $M W(T+\Delta T)-M W(T)$, for different values of $\Delta T$ (that is, ramp definition 1) and study the statistical properties of these derived data. For example, Figure 4 shows the change in wind generation over 30 minute intervals for 2008. At each 5 minute time interval, the y-axis is the difference between the generation at that time interval and 30 minutes later. A drop in generation is indicated by negative values. We consider changes of all magnitudes in our analysis; extreme events are not removed as we want to obtain statistics on such events as well.

When we consider similar plots for 15 minute and 60 minute intervals, the changes in wind generation are smaller and larger, respectively, relative to the 30 minute differences, as shown in Table I. This table lists how many data points (each 5 min interval is considered one data point) are the start of a change of a given magnitude, for a given $\Delta T$. We do observe that if the generation is stable at a certain value and then suddenly drops, stabilizing at a lower value, then many data points around the time of the drop will exhibit a large change.

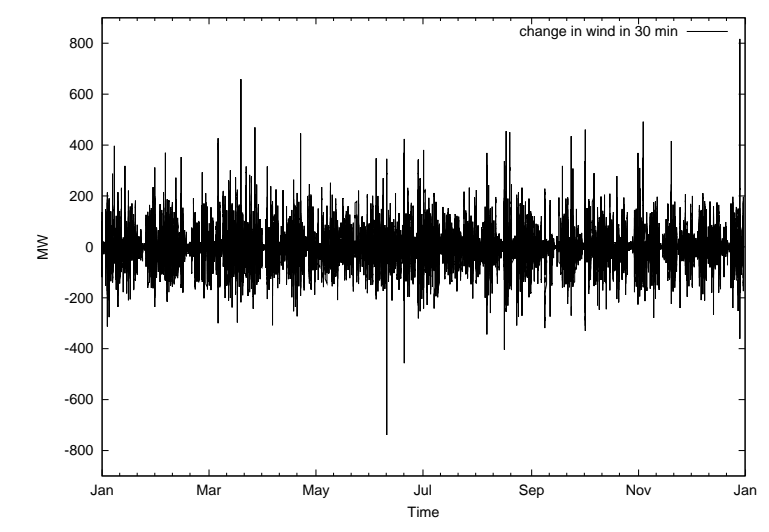

Fig. 4. The change in wind energy generated for $30 \mathrm{~min}$ intervals (ramp definition 1).

For example, if the generation is $1000 \mathrm{MW}$ at times $\mathrm{T}, \mathrm{T}+1$, $\mathrm{T}+2$, and drops to $500 \mathrm{~mW}$ at times $\mathrm{T}+3, \mathrm{~T}+4$, and $\mathrm{T}+5$, then, if we consider 15 minute changes in generation, each of the intervals, $\mathrm{T}, \mathrm{T}+1$, and $\mathrm{T}+2$ will have a $500 \mathrm{MW}$ drop. This fact is illustrated in Table I, where a single large drop of 725 MW in one 5 minute interval, appears thrice when 15 minute changes are considered.

A similar summary for ramp definition 2 , based on the maximum and minimum energy in an interval, is presented in Table II. Tables I and II indicate that for any time interval $\Delta T$, at low values of $|\Delta M W|$ (say less than $50 \mathrm{MW}$ ), the number of data points which start a negative change of that magnitude is larger than the number which start a positive change. However, for larger values of $|\Delta M W|$, which are essentially the ramp events, the number of positive changes is larger than the number of negative changes. In other words, we expect more intervals which are the start of a positive ramps than intervals which are the start of a negative ramp. In addition, we observe that the negative ramps tend to be less severe than the positive ones.

Table III lists the minimum and maximum changes, and the times when they occur, for 5, 15, 30, and 60 minute intervals, for the two definitions. We have included the 5 minute interval to show that large increases and decreases can occur between consecutive time intervals (recall that the data are at 5 minute intervals). The large negative drop on June 11 within a 5 minute interval is sufficiently large that it determines the time of the largest negative ramp for the 15,30 , and 60 minute intervals as well.

Tables I - III show that the statistics obtained for the change in generation over various time intervals are not very different between the two definitions. For smaller $\Delta T$, the results from the two are very similar. For larger $\Delta T$, as we expect, definition 2 identifies more intervals as the start of a large change in energy. These additional intervals are the ones where there is a large change in energy over, say, a 60 minute interval, but the generation at the two end points are close to each other. Such intervals appear in the low $|\Delta M W|$ rows in Table I for definition 1. Other than this expected change, the two tables are qualitatively similar.

Next, we consider the time of day when the ramp events 


\begin{tabular}{c|c|c|c|c|c|c|c|c}
\hline$|\Delta M W|$ & \multicolumn{2}{|c|}{5 minute } & \multicolumn{2}{c|}{15 minute } & \multicolumn{2}{c|}{30 minute } & \multicolumn{2}{c}{60 minute } \\
& pos & neg & pos & neg & pos & neg & pos & neg \\
\hline$=0$ & 10574 & - & 6378 & - & 4746 & - & 3522 & - \\
$>0 M W,<50 M W$ & 45686 & 48353 & 42162 & 45464 & 35573 & 38325 & 28065 & 30341 \\
$\geq 50 M W,<100 M W$ & 409 & 274 & 4930 & 4906 & 8906 & 9897 & 10787 & 11933 \\
$\geq 100 M W,<200 M W$ & 48 & 20 & 855 & 556 & 3731 & 3428 & 7547 & 8628 \\
$\geq 200 M W,<500 M W$ & 2 & 6 & 85 & 32 & 488 & 248 & 2573 & 1846 \\
$\geq 200 M W,<500 M W$ & 0 & 1 & 0 & 3 & 10 & 6 & 89 & 27 \\
$\geq 1000 M W$ & 0 & 0 & 0 & 0 & 0 & 0 & 4 & 0 \\
\hline
\end{tabular}

TABLE I

DISTRIBUTION OF CHANGES IN WIND GENERATION (DEFINITION 1) FOR DIFFERENT VALUES OF $\Delta T$.

\begin{tabular}{c|c|c|c|c|c|c}
\hline & \multicolumn{2}{|c|}{15 minute } & \multicolumn{2}{c|}{30 minute } & \multicolumn{2}{c}{60 minute } \\
& pos & neg & pos & neg & pos & neg \\
\hline$=0$ & 3447 & - & 2442 & - & 1745 & - \\
$>0 M W,<50 M W$ & 43338 & 46448 & 33924 & 36529 & 21894 & 23444 \\
$\geq 50 M W,<100 M W$ & 5235 & 5245 & 11363 & 12255 & 15095 & 15992 \\
$\geq 100 M W,<200 M W$ & 889 & 584 & 4172 & 3819 & 10241 & 11464 \\
$\geq 200 M W,<500 M W$ & 91 & 36 & 547 & 292 & 3072 & 2288 \\
$\geq 200 M W,<500 M W$ & 0 & 3 & 10 & 6 & 97 & 29 \\
$\geq 1000 M W$ & 0 & 0 & 0 & 0 & 4 & 0 \\
\hline
\end{tabular}

TABLE II

DiSTRIBUTION OF CHANGES IN WIND GENERATION (DEFINITION 2) FOR DIFFERENT VALUES OF $\Delta T$. THE 5 MINUTE CHANGE IS NOT INCLUDED AS IT IS THE SAME AS IN TABLE I

\begin{tabular}{c|c|c|c|c} 
& $5 \mathrm{~min}$ & $15 \mathrm{~min}$ & $30 \mathrm{~min}$ & $60 \mathrm{~min}$ \\
\hline Maximum & 304 & 460 & 817 & 1057 \\
At time & $10 / 2,8: 00$ & $12 / 2910: 00$ & $12 / 299: 55$ & $12 / 299: 50$ \\
\hline Minimum & -725 & -734 & $-739(-741)$ & $-728(-741)$ \\
At time & $6 / 11 \quad 12: 35$ & $6 / 1112: 20$ & $6 / 1112: 20$ & $6 / 1111: 50$ \\
\hline
\end{tabular}

TABLE III

MiNIMUM AND MAXIMUM VALUES FOR THE CHANGES IN WIND ENERGY GENERATION FOR DIFFERENT INTERVALS. VALUES FOR THE SECOND DEFINITION ARE LISTED IN PARENTHESIS ONLY WHEN THEY ARE DIFFERENT FROM THE VALUE FOR DEFINITION 1

occur. For this, we need to select the threshold defining the ramp; any time interval which includes a change larger in magnitude than this threshold is considered to be the start of a ramp event. Focusing on the 15, 30, and 60 minute time intervals, we select three thresholds for each, to identify ramps of low, moderate, and high severity. We consider each day to be divided into four parts: early and late morning, and early and late afternoon, representing the times from midnight-6:00am, 6:00am-noon, noon-6:00pm, and 6:00pm midnight, respectively. Then, considering the changes in the wind generation for the three time intervals, $\Delta T=15,30$, and 60 minutes, we collect statistics on when ramp events exceeding a certain magnitude occurred.

For a certain time of day, say early morning, there are two ways in which we can count the ramp events occurring during this time period:

- We can explicitly count all the intervals which start a ramp event during this period. This can be considered as over-counting as a ramp event which occurs over a long time will have several intervals which "start" the event. Also, as mentioned earlier, a large drop in generation between two consecutive intervals can result in several data points meeting the ramp threshold criterion.

- Alternatively, we can do a binary count where a time period is considered to have a ramp event if there is at least one event which starts during this time period. This could be considered as under counting the number of ramps in a time period as many distinct ramps occurring during this period will not be individually counted.

We tried both approaches and while the counts of ramp events are different, the resulting conclusions are similar. In this paper, we present the results of the second approach, where the count can be considered as the number of days in the year in which a positive or negative ramp of magnitude greater than the threshold starts in that time period of the day. However, if a ramp event straddles a time period boundary, it can get counted in two time periods of a day. Tables IV-VI show these counts for positive and negative ramps at 15,30 , and 60 minute intervals, defined using various threshold levels, indicating low, moderate, and high ramp severity levels. Values for ramp definition 2 are included in parenthesis whenever they are different from values for ramp definition 1 .

These tables indicate that while both positive and negative ramps of any magnitude can occur at any time of the day, there are times when they are more likely. For example, we 


\begin{tabular}{ccccc}
\hline & & $\mathrm{Tr}_{l}=150$ & $\mathrm{Tr}_{m}=250$ & $\mathrm{Tr}_{h}=350$ \\
\hline \multirow{2}{*}{ Early am } & pos & 5 & 2 & 1 \\
& neg & 7 & 0 & 0 \\
\hline \multirow{2}{*}{ Late am } & pos & 12 & 5 & 1 \\
& neg & 6 & 1 & 0 \\
\hline \multirow{2}{*}{ Early pm } & pos & 28 & 3 & 1 \\
& neg & 16 & 4 & 2 \\
\hline \multirow{2}{*}{ Late pm } & pos & 19 & 5 & 1 \\
& neg & 16 & 3 & 1 \\
\hline Total & pos & 64 & 15 & 4 \\
& neg & 45 & 8 & 3 \\
\hline
\end{tabular}

TABLE IV

TIME OF DAY OCCURRENCES OF 15MIN RAMPS, AT LOW, MODERATE, AND HIGH THRESHOLDS. THE NUMBER INDICATES THE NUMBER OF DAYS IN THE YEAR IN WHICH A POSITIVE OR NEGATIVE RAMP OF MAGNITUDE GREATER THAN THE THRESHOLD START IN THAT TIME PERIOD OF THE DAY. VALUES FOR THE TWO RAMP DEFINITIONS ARE IDENTICAL.

\begin{tabular}{ccccc}
\hline & & $\operatorname{Tr}_{l}=200$ & $\operatorname{Tr}_{m}=300$ & $\operatorname{Tr}_{h}=400$ \\
\hline Early am & pos & $9(10)$ & 2 & 2 \\
& neg & 16 & 0 & 0 \\
\hline \multirow{2}{*}{ Late am } & pos & 19 & 7 & 4 \\
& neg & $11(12)$ & 2 & 0 \\
\hline \multirow{2}{*}{ Early pm } & pos & $52(53)$ & 13 & 2 \\
& neg & $22(24)$ & 5 & 2 \\
\hline \multirow{2}{*}{ Late pm } & pos & $25(27)$ & 11 & 4 \\
& neg & 23 & $4(5)$ & 1 \\
\hline Total & pos & $105(109)$ & 33 & 12 \\
& neg & $72(75)$ & $11(12)$ & 3 \\
\hline
\end{tabular}

TABLE V

TIME OF DAY OCCURRENCES OF 30 MIN RAMPS, AT LOW, MODERATE, AND HIGH THRESHOLDS. THE NUMBER INDICATES THE NUMBER OF DAYS IN THE YEAR IN WHICH A POSITIVE OR NEGATIVE RAMP OF MAGNITUDE GREATER THAN THE THRESHOLD STARTS IN THAT TIME PERIOD OF THE DAY. VALUES FOR RAMP DEFINITION 2 ARE LISTED IN PARENTHESIS WHENEVER THEY ARE DIFFERENT FROM VALUES FOR DEFINITION 1.

\begin{tabular}{ccccc}
\hline & & $\mathrm{Tr}_{l}=300$ & $\mathrm{Tr}_{m}=450$ & $\mathrm{Tr}_{h}=600$ \\
\hline Early am & pos & 9 & 3 & 1 \\
& neg & 16 & 0 & 0 \\
\hline Late am & pos & $22(23)$ & 4 & 2 \\
& neg & $6(8)$ & 1 & 1 \\
\hline \multirow{2}{*}{ Early pm } & pos & $50(52)$ & 7 & 2 \\
& neg & 16 & 4 & 2 \\
\hline Late pm & pos & 27 & 8 & 3 \\
& neg & $18(20)$ & 3 & 0 \\
\hline Total & pos & $108(111)$ & 22 & 8 \\
& neg & $56(60)$ & 8 & 3 \\
\hline
\end{tabular}

TABLE VI

TIME OF DAY OCCURRENCES OF 60 MIN RAMPS, AT LOW, MODERATE, AND HIGH THRESHOLDS. THE NUMBER INDICATES THE NUMBER OF DAYS IN THE YEAR IN WHICH A POSITIVE OR NEGATIVE RAMP OF MAGNITUDE GREATER THAN THE THRESHOLD STARTS IN THAT TIME PERIOD OF THE DAY. VALUES FOR RAMP DEFINITION 2 ARE LISTED IN PARENTHESIS WHENEVER THEY ARE DIFFERENT FROM VALUES FOR DEFINITION 1 observe that moderate to large downward ramps rarely occur during early or late morning. The single negative ramp event at high threshold in Table VI in the late morning is due to the large drop of $-725 \mathrm{MW}$ which occurs just after noon on June 11, 2008 (see Table III). We also observe that positive ramps occur more frequently in the afternoon than in the morning. These data also confirm that there are more days with positive ramps than with negative ramps. Both positive and negative ramps, of moderate to large severity, can occur on the same day, as shown in Figure 3.

We observe that the choice of a ramp definition has little effect on the results of the time of day occurrence of ramp events. The changes are minor and mostly at the low thresholds. As expected, ramp definition 2 will identify more ramp events, which is what we see in the results thus far.

Finally, we analyze the ramp events to determine if there are any monthly variations. Table VII shows the number of days in a month when ramps with low to moderate severity occur. A day may have more than one ramp with the given level of severity, but this is not reflected in the table. An analysis of each row indicates that there are some months (March and August) when there is greater probability of ramp events. However, there is too little data in this table to draw any strong conclusions, especially as it reflects trends for a single year. Again, there is little difference in the results between the two ramp definitions.

\section{CONCLUSION}

In this paper, we have shown that simple statistical analysis of wind generation can provide insights into ramp events such as distributions of their severity levels, their time of occurrence during the day, and their occurrence by month. While the results of such analysis will be different depending on the location of the wind farm and possibly the amount of wind generation, it can none-the-less provide grid operators additional information they can use in balancing the load.

We considered two ways of defining the change in wind energy generation in a time interval - one approach focused just on the end points, while the other considered the minimum and maximum generation between the two end points. Our analysis showed that the results did not differ between these two definitions.

We also categorized ramps based on different thresholds low, moderate, and high. Our analysis using data from BPA showed that moderate to large downward ramps rarely occurred in early to late morning, while positive ramps occurred more frequently in the afternoons than in the mornings. In addition, certain months of the year had more occurrences of ramps than others.

We will next extend this work by repeating the anlysis using wind generation data from another region as well as 2009 data for the Columbia Basin region. In addition, we will explore associations of ramp events with weather conditions to determine if we can identify days likely to have ramps.

\section{ACKNOWLEDGMENT}

This work was funded through the Renewable System Interconnect Program in the Office of Energy Efficiency and 


\begin{tabular}{llllcccccccccc}
\hline & & $\mathrm{J}$ & $\mathrm{F}$ & $\mathrm{M}$ & $\mathrm{A}$ & $\mathrm{M}$ & $\mathrm{J}$ & $\mathrm{J}$ & $\mathrm{A}$ & $\mathrm{S}$ & $\mathrm{O}$ & $\mathrm{N}$ & $\mathrm{D}$ \\
\hline \multirow{2}{*}{ 15min, $\mathrm{Tr}=150$} & pos & 5 & 6 & 10 & 5 & 1 & 7 & 1 & 7 & 3 & 3 & 4 & 2 \\
& neg & 4 & 1 & 5 & 4 & 0 & 5 & 2 & $8(9)$ & 2 & 5 & 1 & 2 \\
$30 \mathrm{~min}, \mathrm{Tr}=200$ & pos & 9 & $6(7)$ & 12 & 9 & 5 & 9 & 5 & 10 & 4 & 5 & 5 & 2 \\
& neg & 6 & 2 & $8(9)$ & 5 & 1 & $5(7)$ & 5 & $8(9)$ & 4 & 6 & 6 & 6 \\
60min, Tr=300 & pos & 9 & 7 & 12 & $5(6)$ & 6 & 9 & 6 & 10 & 7 & 6 & 6 & 5 \\
& neg & 3 & 1 & 5 & 5 & 1 & $5(6)$ & 1 & 8 & 6 & 4 & 6 & 3 \\
15min, Tr=250 & pos & 0 & 0 & 2 & 1 & 0 & 2 & 0 & 3 & 1 & 1 & 3 & 1 \\
& neg & 0 & 0 & 0 & 0 & 0 & 3 & 0 & 2 & 0 & 1 & 0 & 1 \\
$30 \mathrm{~min}, \mathrm{Tr}=300$ & pos & 3 & 2 & 5 & 2 & 0 & 4 & 1 & 4 & 3 & 1 & 4 & 1 \\
& neg & 1 & 0 & 0 & 1 & 0 & 2 & 0 & 3 & 1 & 1 & 0 & 1 \\
& pos & 1 & 2 & 3 & 1 & 0 & 2 & 1 & 2 & 3 & 3 & 1 & 1 \\
& neg & 0 & 0 & 1 & 0 & 0 & 2 & 0 & 1 & 1 & 1 & 0 & 1 \\
\hline
\end{tabular}

TABLE VII

NUMBER OF DAYS WITH RAMP EVENTS IN EACH MONTH. THE COLUMNS ARE THE MONTHS AND THE ROWS ARE THE RAMPS FOR 15,30 , AND 60 MINUTE INTERVALS. LOW SEVERITY RAMPS ARE LISTED BEFORE THE MODERATE SEVERITY RAMPS. VALUES FOR RAMP DEFINITION 2 ARE LISTED IN PARENTHESIS WHENEVER THEY ARE DIFFERENT FROM VALUES FOR DEFINITION 1.

Renewable Energy at the US Department of Energy.

This work performed under the auspices of the U.S. Department of Energy by Lawrence Livermore National Laboratory under Contract DE-AC52-07NA27344.

\section{REFERENCES}

[1] D. Hawkins and M. Rothleder, "Evolving role of wind forecasting in market operation at the CaISO," in Proceedings, IEEE Power Systems Conference and Exposition, 2006, pp. 234-238. [Online]. Available: DOI:10.1109/PSCE.2006.296304

[2] "How BPA supports wind power in the pacific northwest," Bonneville Power Administration Fact Sheet. [Online]. Available: http://www.bpa.gov/corporate/pubs/fact_sheets/09fs/BPA_supports_ wind_power_for_the_Pacific_Northwest_-_Mar_2009.pdf

[3] "Balancing act: BPA grid responds to huge influx of wind power," Bonneville Power Administration Fact Sheet. [Online]. Available: http://www.bpa.gov/corporate/pubs/fact_sheets/08fs/ Wind-Balancing-act-Nov2008.pdf
[4] "BPA wind projects map: Current and proposed wind project interconnections to BPA transmission facilities." [Online]. Available: http://www.transmission.bpa.gov/PlanProj/Wind/documents/ map-BPA_wind_interconnections.pdf

[5] U. Focken and M. Lange, "Wind power forecasting pilot project in Alberta, Canada: Final Report," Energy and Meteo Systems, GmbH, Tech. Rep., May 2008. [Online]. Available: www.uwig.org/Final_report_ emsys_lv.pdf

[6] J. H. Pease, Private communication, Bonneville Power Administration, July 2009.

Chandrika Kamath Chandrika Kamath is a researcher at Lawrence Livermore National Laboratory, where she is involved in the analysis of data from scientific simulations, observations, and experiments. She received her Ph.D. in Computer Science from the University of Illinois at Urbana-Champaign. Her research interests include signal and image processing, machine learning, pattern recognition, and statistics, as well as the application of data mining techniques to the solution of practical problems. 